\title{
Asymmetric rotating array beams with free movement and revolution
}

\author{
Jia Xu (胥 佳), Zhenglin Liu (刘政麟), Keming Pan (潘柯铭), and Daomu Zhao (赵道木) \\ Zhejiang Provincial Key Laboratory of Quantum Technology and Device, Department of Physics, Zhejiang University, Hangzhou 310027, China \\ *Corresponding author: zhaodaomu@yahoo.com \\ Received October 13, 2021 | Accepted December 2, 2021 | Posted Online January 4, 2022
}

\begin{abstract}
We introduce a new class of partially coherent asymmetric array beams. When the beam propagates, the spectral density of each lobe and the corresponding degree of coherence have rotating behavior. Especially, not only can array-like lattices revolve arbitrarily, but also they can move freely by controlling transverse plane shifts. Furthermore, we have generated this kind of beam experimentally, and the experimental phenomena are consistent with the numerical simulation results. Such a rotating beam with free movement and revolution may broaden the way for optical applications. More importantly, it inspires further studies in the field of asymmetric coherence gratings and lattices.
\end{abstract}

\begin{abstract}
Keywords: partial coherence; asymmetric array beams; rotating behavior; transverse plane shifts; free movement and revolution.

DOI: 10.3788/COL202220.022602
\end{abstract}

\section{Introduction}

In the space-frequency formulation of the coherence theory of stationary optical fields, the cross-spectral density (CSD) function superimposed by the coherent propagation mode has attracted wide attention. To gain some insight about properties of fields radiated by sources of different states of coherence, the Gaussian Schell model (GSM) source is widely used, which can bring simpler analysis results for the non-trivial incident field and media of the beam interaction ${ }^{[1]}$. Recent years have witnessed significant progress in the characteristics of the beams, for instance, flap-top beams ${ }^{[2]}$, twisted array beams ${ }^{[3]}$, and hollow beams ${ }^{[4]}$. At the same time, there are continuous breakthroughs in experiments and simulations ${ }^{[5,6]}$.

In order to design a novel beam field, the conditions of the amplitude and phase of the complex coherence in one and two dimensions are derived theoretically ${ }^{[7,8]}$. Further analysis shows the special effects of the Cartesian phase on the lateral shifting of the light source ${ }^{[9]}$. The Cartesian counterpart can realize the separation in the $x$ and $y$ coordinates ${ }^{[10]}$. A specific weighting factor is assigned to the linear phase and it is then linearly superimposed. In this way, a periodic structure of source degree of coherence (DOC) can be realized, thereby forming a grating-like and lattice-like radiation pattern ${ }^{[11]}$. This has been verified analytically and experimentally when generating an asymmetric lattice ${ }^{[12]}$. It should also be mentioned that there is already a combination of vortex phase and linear Cartesian phase, and there is no superposition of rotational phase and Cartesian phase. Compared with the former, the latter will have lobe rotation and overall revolution. In addition, it is well known that the initial introduction of the twisted phase is due to the study of the rotationally invariant CSD function ${ }^{[13]}$. Twisted GSMs are due to the presence of this type of phase term in the GSM source. Distorted partially coherent beams have also made some important conceptual developments in recent years ${ }^{[14-17]}$. In order to achieve larger degrees of freedom and flexibility in the beam twist, several types of typical partially coherent beams have been introduced recently ${ }^{[18,19]}$. The sign of the propagation process is that the spectral density and coherence tend to rotate. Then, by combining the spatial light modulator (SLM) with the astigmatic phase, this type of rotating beam was also obtained experimentally ${ }^{[6]}$.

Here, we show a type of random source whose CSD combines the lobe rotation, the beam revolution, and linear shifts of the array. We establish such a beam and then explore its process of propagation as a controllable two-dimensional optical array that carries rotating and revolving structures. It is noteworthy that, while the rotating phase is capable of producing rotating beams upon propagation, the linear Cartesian phase is responsible for splitting beams, obtaining a series of replicas and then forming the asymmetric array according to the shifting of different weights.

\section{Theory}

Let us first review the main theoretical descriptions related to stationary beams. Suppose that a scalar random beam-like field 
is generated from a planar source located in the plane of $z=0$ and then propagated in the direction of positive $z$. In optical coherence theory ${ }^{[20]}$, the second-order statistic of the field is expressed as the CSD function of two points $r_{1}$ and $r_{2}$ in the source plane:

$$
W^{(0)}\left(r_{1}, r_{2} ; \omega\right)=\left\langle U^{*}\left(r_{1} ; \omega\right) U\left(r_{2} ; \omega\right)\right\rangle,
$$

where $U$ represents the fluctuating field in the source plane, and angle brackets represent the average value of the whole. The asterisk signifies the complex conjugate. For the sake of brevity, the angular frequency dependence of all of the quantities of interest will be omitted but implied in the following. To be a genuine correlation function in mathematics, the CSD must correspond to a non-negative definite kernel. The kernel is satisfied if the CSD function can be written as the superposition integral of the following form ${ }^{[1]}$ :

$$
W^{(0)}\left(r_{1}, r_{2}\right)=\int p(v) H_{0}^{*}\left(r_{1}, v\right) H_{0}\left(r_{2}, v\right) \mathrm{d}^{2} v,
$$

where $p(v)$ is an arbitrary non-negation weight function for any $v$, and $H_{0}(r, v)$ is an arbitrary or even possibly complex-valued kernel.

We will first illustrate how the rotating array spectral density can be obtained directly. An anisotropic Gaussian correlated field with linear shifting parameters $a_{s}$ is required ${ }^{[11]}$,

$$
\begin{aligned}
p\left(v_{x}, v_{y}\right)= & \frac{4 \pi \delta_{x} \delta_{y}}{C_{x} C_{y}} \sum_{n_{x}=1}^{N_{x}} \sum_{n_{y}=1}^{N_{y}}\left\{\exp \left[-\delta_{x}^{2}\left(2 \pi v_{x}+a_{x}\right)^{2}\right]\right. \\
& \left.\times \exp \left[-\delta_{y}^{2}\left(2 \pi v_{y}+a_{y}\right)^{2}\right]\right\},
\end{aligned}
$$

where $\delta_{s}(s=x, y)$ are positive real constants whose values are related to the spatial coherence widths along the $x$ and $y$ directions. $C_{s}=\sum_{n_{s}=1}^{N_{s}}$ are the normalization factor. $a_{s}=\eta_{s} N_{s}$ and $\eta_{s}$ are any real numbers. Then, $\eta_{s}$ and $N_{s}$ can determine the coherence state of the source and the spectral density of the far field.

$H_{0}$ uses the following form to generate a light source with a partially coherent beam with the characteristics of revolution and rotation:

$$
\begin{aligned}
H_{0}(r, v)= & \tau(r) \exp \left[-2 \pi i v_{x}(x \cos \alpha-y \sin \alpha)\right] \\
& \times \exp \left[-2 \pi i v_{y}(x \sin \alpha+y \cos \alpha)\right] \\
& \times \exp [i u(x \cos \theta-y \sin \theta)(x \sin \theta+y \cos \theta)],
\end{aligned}
$$

where $u$ is the beam twist parameter, which is the real constant. The arbitrary angles $\alpha$ and $\theta$ characterize the revolution of the overall structure around the beam axis and the rotation of phase structure around every lobe axis, respectively, which are performed by rotation matrices $\left(\begin{array}{cc}\cos \alpha & -\sin \alpha \\ \sin \alpha & \cos \alpha\end{array}\right)$ and $\left(\begin{array}{cc}\cos \theta & -\sin \theta \\ \sin \theta & \cos \theta\end{array}\right)$. The amplitude profile function $\tau(r)$ here takes the form of an anisotropic Gaussian profile:

$$
\tau(r)=\exp \left(-\frac{x^{2}}{4 \sigma_{x}^{2}}\right) \exp \left(-\frac{y^{2}}{4 \sigma_{y}^{2}}\right)
$$

where $\sigma_{x}$ and $\sigma_{y}$ are the spectral density widths along the $x$ and $y$ directions in the source plane, respectively. On substituting Eqs. (4) and (3) into Eq. (2), the CSD function is expressed as

$$
\begin{aligned}
W^{(0)}\left(r_{1}, r_{2}\right)= & \frac{1}{C_{x} C_{y}} \sum_{n_{x}}^{N_{x}} \sum_{n_{y}=1}^{N_{y}}\left\{\exp \left(-\frac{x_{1}^{2}+x_{2}^{2}}{4 \sigma_{x}^{2}}-\frac{y_{1}^{2}+y_{2}^{2}}{4 \sigma_{y}^{2}}\right)\right. \\
& \times \exp \left[\left(\frac{\sin 2 \alpha}{4 \delta_{x}^{2}}-\frac{\sin 2 \alpha}{4 \delta_{y}^{2}}\right)\left(x_{2}-x_{1}\right)\left(y_{2}-y_{1}\right)\right] \\
& \times \exp \left[-\left(\frac{\cos ^{2} \alpha}{4 \delta_{x}^{2}}+\frac{\sin ^{2} \alpha}{4 \delta_{y}^{2}}\right)\left(x_{2}-x_{1}\right)^{2}\right] \\
& \times \exp \left[-\left(\frac{\sin ^{2} \alpha}{4 \delta_{x}^{2}}+\frac{\cos ^{2} \alpha}{4 \delta_{y}^{2}}\right)\left(y_{2}-y_{1}\right)^{2}\right] \\
& \times \exp \left[i\left(a_{x} \cos \alpha+a_{y} \sin \alpha\right)\left(x_{2}-x_{1}\right)\right. \\
& +i\left(a_{y} \cos \alpha-a_{x} \sin \alpha\right)\left(y_{2}-y_{1}\right) \\
& +\frac{i u \sin 2 \theta}{2}\left(x_{2}^{2}-x_{1}^{2}+y_{1}^{2}-y_{2}^{2}\right) \\
& \left.\left.+\frac{i u \cos 2 \theta}{2}\left(x_{2} y_{2}-x_{1} y_{1}\right)\right]\right\} .
\end{aligned}
$$

According to the generalized Huygens-Fresnel principle, the $\mathrm{CSD}$ that is propagated by the optical $\mathrm{ABCD}$ system in the transverse plane $z>0$ is characterized by ${ }^{[21,22]}$

$$
\begin{aligned}
W\left(\rho_{1}, \rho_{2}, z\right)= & \frac{k^{2}}{4 B^{2} C_{x} C_{y}} \sqrt{\frac{1}{a_{x s} a_{y s} T_{1} \varepsilon}} \\
& \times \sum_{n_{x}=1}^{N_{x}} \sum_{n_{y}=1}^{N_{y}}\left\{\exp \left[-\frac{i k D}{2 B}\left(\rho_{1}^{2}-\rho_{2}^{2}\right)\right]\right. \\
& \times \exp \left[\frac{-k^{2}\left(\rho_{1 x}-\rho_{2 x}\right)^{2}}{4 a_{x s} B^{2}}+\frac{-k^{2}\left(\rho_{1 y}-\rho_{2 y}\right)^{2}}{4 a_{y s} B^{2}}\right] \\
& \times \exp \left(-\frac{\Lambda_{x}^{2}}{4 T_{1}}+\frac{\Omega_{1}^{2}}{4 T_{1}}+\frac{i \Lambda_{x} \Omega_{1}}{2 T_{1}}\right) \\
& \left.\times \exp \left[\frac{1}{4 \varepsilon}\left(\Omega_{2}+i \Lambda_{y}+\frac{i \Lambda_{x} G}{2 T_{1}}+\frac{\Omega_{1} G}{2 T_{1}}\right)^{2}\right]\right\},
\end{aligned}
$$

where 
$a_{x s}=\frac{1}{2 \sigma_{x}^{2}} ; \quad a_{y s}=\frac{1}{2 \sigma_{y}^{2}} ; \quad \gamma=u \cos 2 \theta$

$\beta_{1}=\frac{k A}{B}+u \sin 2 \theta ; \quad \beta_{2}=\frac{k A}{B}-u \sin 2 \theta ;$

$a_{n x}=a_{x} \cos \alpha+a_{y} \sin \alpha ; \quad a_{n y}=-a_{x} \sin \alpha+a_{y} \cos \alpha ;$

$T_{1}=\frac{\cos ^{2} \alpha}{4 \delta_{x}^{2}}+\frac{\sin ^{2} \alpha}{4 \delta_{y}^{2}}+\frac{1}{8 \sigma_{x}^{2}}+\frac{\beta_{1}^{2}}{4 a_{x s}}+\frac{\gamma^{2}}{4 a_{y s}}$;

$T_{2}=\frac{\sin ^{2} \alpha}{4 \delta_{x}^{2}}+\frac{\cos ^{2} \alpha}{4 \delta_{y}^{2}}+\frac{1}{8 \sigma_{y}^{2}}+\frac{\beta_{2}^{2}}{4 a_{y s}}+\frac{\gamma^{2}}{4 a_{x s}}$;

$G=-\left(\frac{\beta_{1}}{a_{x s}}+\frac{\beta_{2}}{a_{y s}}\right) \frac{\gamma}{2}+\left(\frac{1}{4 \delta_{x}^{2}}-\frac{1}{4 \delta_{y}^{2}}\right) \sin 2 \alpha ;$

$\varepsilon=T_{2}-\frac{G^{2}}{4 T_{1}}$

$\Omega_{1}=\frac{k \beta_{1}\left(\rho_{2 x}-\rho_{1 x}\right)}{2 a_{x s} B}+\frac{k \gamma\left(\rho_{2 y}-\rho_{1 y}\right)}{2 a_{y s} B} ;$

$\Omega_{2}=\frac{k \gamma\left(\rho_{2 x}-\rho_{1 x}\right)}{2 a_{x s} B}+\frac{k \beta_{2}\left(\rho_{2 y}-\rho_{1 y}\right)}{2 a_{y s} B} ;$

$\Lambda_{x}=a_{n x}-\frac{k\left(\rho_{1 x}+\rho_{2 x}\right)}{2 B} ; \quad \Lambda_{y}=a_{n y}-\frac{k\left(\rho_{1 y}+\rho_{2 y}\right)}{2 B}$

Here, $k=2 \pi / \lambda$ denotes the wavenumber, and $\lambda$ is the optical wavelength in free space. $\rho=\left(\rho_{x}, \rho_{y}\right)$ is the position vector in the output plane. Now, set the same position coordinate in the CSD formula to obtain the spectral density during propagation, $S(\rho, z)=W(\rho, \rho, z)$, and the DOC of two symmetric points with respect to the optical axis, $\mu(\rho,-\rho)=W(\rho,-\rho) /$ $[S(\rho) S(-\rho)]^{1 / 2}$.

Next, in order to better study the morphological characteristics of the beam in the propagation process, we consider adding a lens system, whose transfer matrix can be given by the following formula:

$$
\left[\begin{array}{ll}
A & B \\
C & D
\end{array}\right]=\left[\begin{array}{ll}
1 & z \\
0 & 1
\end{array}\right]\left[\begin{array}{cc}
1 & 0 \\
-\frac{1}{f} & 1
\end{array}\right]=\left[\begin{array}{cc}
1-\frac{z}{f} & z \\
-\frac{1}{f} & 1
\end{array}\right],
$$

where $f=300 \mathrm{~mm}$ is the focal length of the thin lens, and $z$ is the distance between the input plane and the output plane. Since the beam without a lens phase travels at an infinite distance, after adding it, the process of changing the spectral density distribution of the asymmetric rotating GSM array (ARGSMA) beam can be observed within the equivalent focal length.

The propagation characteristics of an ARGSMA beam are depicted in Figs. 1(a)-1(d). Figure 1(a) represents the spectral density of a row of light beams along the propagation direction. Figure 1(b) is the DOC corresponding to Fig. 1(a). The rotation of spectral density is slow first, then fast, and then slow, as seen

(a)

(b)

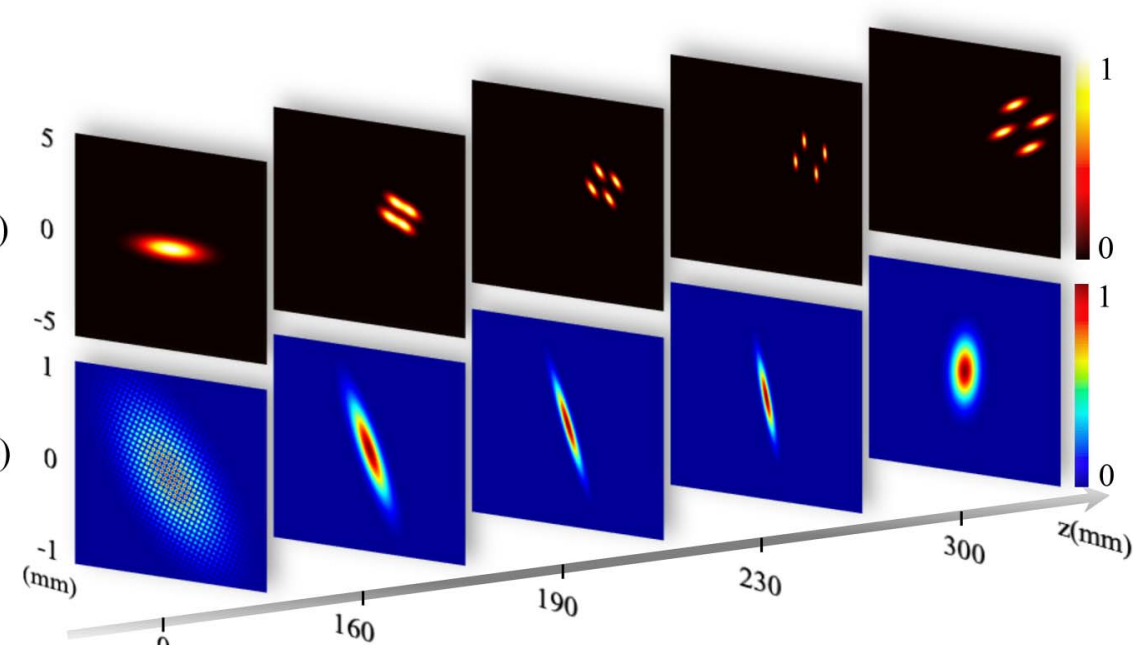

(c)
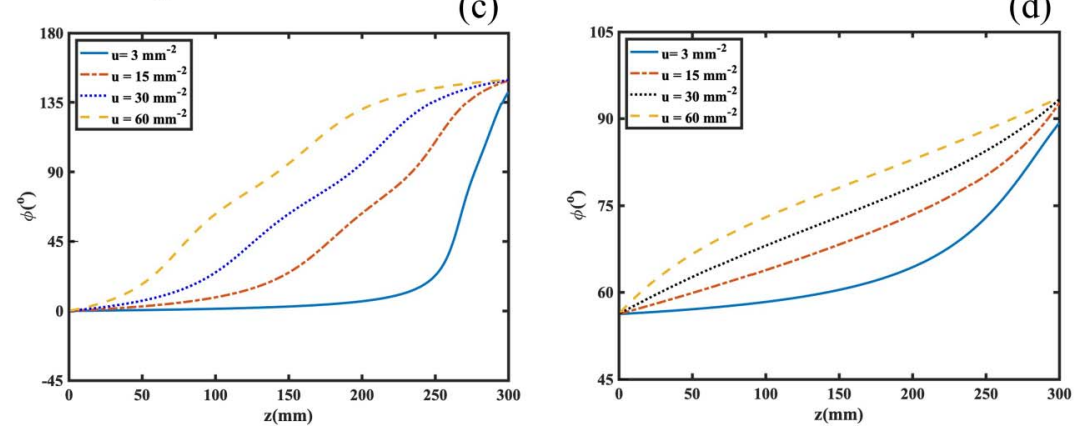

Fig. 1. Propagation dynamics of the beams with (a) spectral density and (b) DOC. Calculated parameters are set as follows: $\mu=15 \mathrm{~mm}^{-2} ; \eta_{x}=\eta_{y}=60 ; N_{x}=N_{y}=2$; $\sigma_{x}=\delta_{x}=1 \mathrm{~mm} ; \sigma_{y}=\delta_{y}=0.3 \mathrm{~mm} ; \alpha=\pi / 8 ; \theta=2 \pi / 3$. Rotation angles versus propagation distance $z$ with $u=3,15,30$, and $60 \mathrm{~mm}^{-2}$ for (c) spectral density and (d) DOC. 
in Fig. 1(c). In order to perfectly represent the shape of each stage of rotation, non-equal spacing is used here to select iconic diagrams. Specifically, five specific positions are given in Figs. 1(a) and $1(\mathrm{~b})$, which are $z=0,160,190,230$, and $300 \mathrm{~mm}$. The initial Gaussian ellipse progressively splits into a rotating $2 \times 2$ Gaussian array upon propagation, which moves along the line at an angle $\alpha=\pi / 8$ to the diagonal shown, as in Fig. 1(a). Each individual lobe of spectral density rotates clockwise all the way around its own central axis in a synchronous motion. The line $\mu=15 \mathrm{~mm}^{-2}$ in Fig. 1(c) also explains the corresponding entire rotation process of spectral density from $z=0 \mathrm{~mm}$ to $300 \mathrm{~mm}$. In the beginning, although the beam can undergo lower speed of a smaller twist, it can speed up and complete a larger

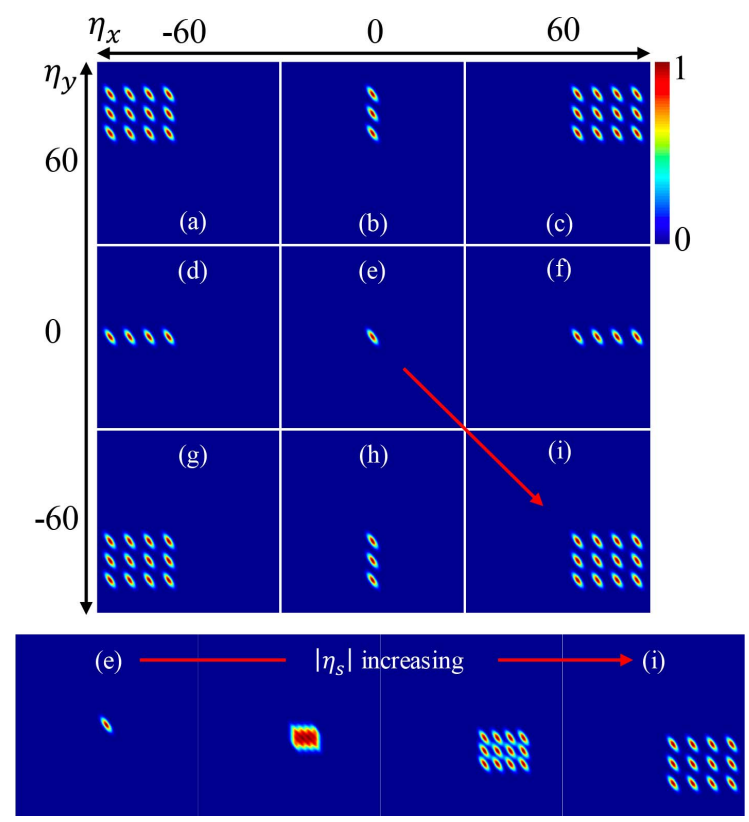

Fig. 2. Free movement of spectral densities with different values of $\eta_{s}$. Calculated parameters are set as follows: $\mu=15 \mathrm{~mm}^{-2} ; z=190 \mathrm{~mm}$; $N_{x}=4 ; N_{y}=3 ; \sigma_{x}=\delta_{x}=1 \mathrm{~mm} ; \sigma_{y}=\delta_{y}=0.3 \mathrm{~mm} ; \alpha=0 ; \theta=2 \pi / 3$. and even a twist of $150 \mathrm{deg}$. The larger the $\mu$ is, the earlier the acceleration starts in Figs. 1(c). Furthermore, Fig. 1(b) presents DOC of the field. It is demonstrated that when the beam propagates, the DOC rotates clockwise around the beam axis and then degenerates into a Gaussian profile. The $\mu$ of different values corresponds to DOC, as shown in Fig. 1(d). In this respect, the rotating array beam can be a potential tool for dynamic control of multiple particles.

Having shown the intriguing property of beams, it is instructive to study the dependency of the light intensity distributions. As clearly seen from Fig. 1(a), the central axis of this beam is not simply along the $z$ axis during propagation, but with the lateral shift. The parameter $\eta_{s}$ determines the amount and direction of the lateral shift, as shown in Fig. 2. Particularly, asymmetric characteristics about the $x$ axis and the $y$ axis depend on negative and positive numbers. It means that when $\eta_{x}=\eta_{y}=60$, the array beam will be moved to the upper right direction. On the contrary, when $\eta_{x}=\eta_{y}=-60$, the array beam will be moved to the bottom left direction. In the same way, when $\eta_{x}=-60$ and $\eta_{y}=60$, the array beam will be moved to the upper left direction. On the contrary, when $\eta_{x}=60$ and $\eta_{y}=-60$, the array beam will be moved to the bottom left direction. The intensity remains symmetric about both axes when $\eta_{x}=\eta_{y}=0$. Meanwhile, it is also possible to achieve only one axial symmetry by changing its number. To develop an understanding of the transverse plane shifts, the specific splitting process of Figs. 2(e) to 2(i) is given above. As the value of $\left|\eta_{s}\right|$ gradually increases, the beam gradually splits and spreads in a certain direction. What is more, it is also noticeable that the value of the parameter $N_{s}$ determines the dimension of the array.

As shown in Fig. 3, it is worth mentioning that the revolution of the array beam and rotation of each lobe is controlled by $\alpha$ and $\theta$, respectively. To be specific, Fig. 3(a) shows that the array beam revolves around the fixed center of the figure and completes a circle, as $\alpha$ increases from 0 to $2 \pi$. Figure 3(b) shows the size of $\theta$ will affect the rotation direction of each lobe. $\pi / 4$ and $3 \pi / 4$ are the special points. At these two points, the lobe

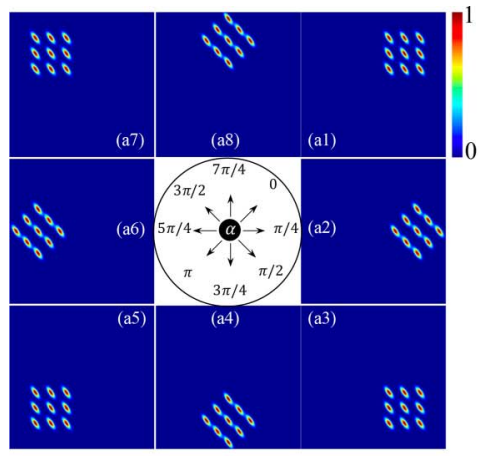

(a)

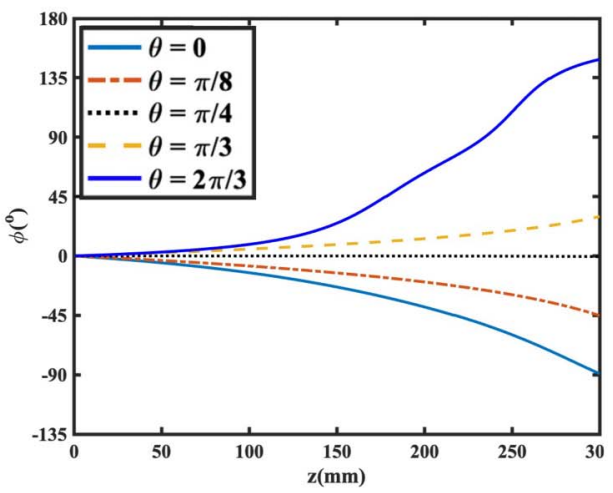

(b)

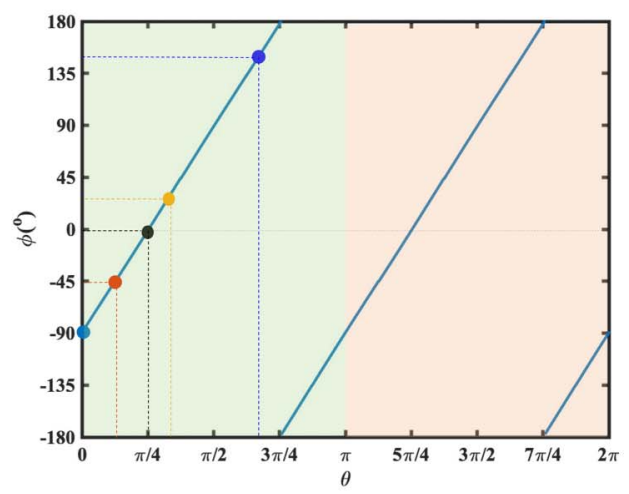

(c)

Fig. 3. (a) Revolution angles of the array beam with different values of $\alpha$. Calculated parameters are set as follows: $\mu=15 \mathrm{~mm}^{-2} ; z=190 \mathrm{~mm} ; \eta_{x}=\eta_{y}=60$; $N_{x}=N_{y}=3 ; \sigma_{x}=\delta_{x}=1 \mathrm{~mm} ; \sigma_{y}=\delta_{y}=0.3 \mathrm{~mm} ; \theta=2 \pi / 3$. (b) Rotation angles of the lobes during transmission with $\theta=0, \pi / 8, \pi / 4, \pi / 3$, and $2 \pi / 3$. (c) The relationship between the rotation angles of the lobes and $\theta$ at $z=f$. 


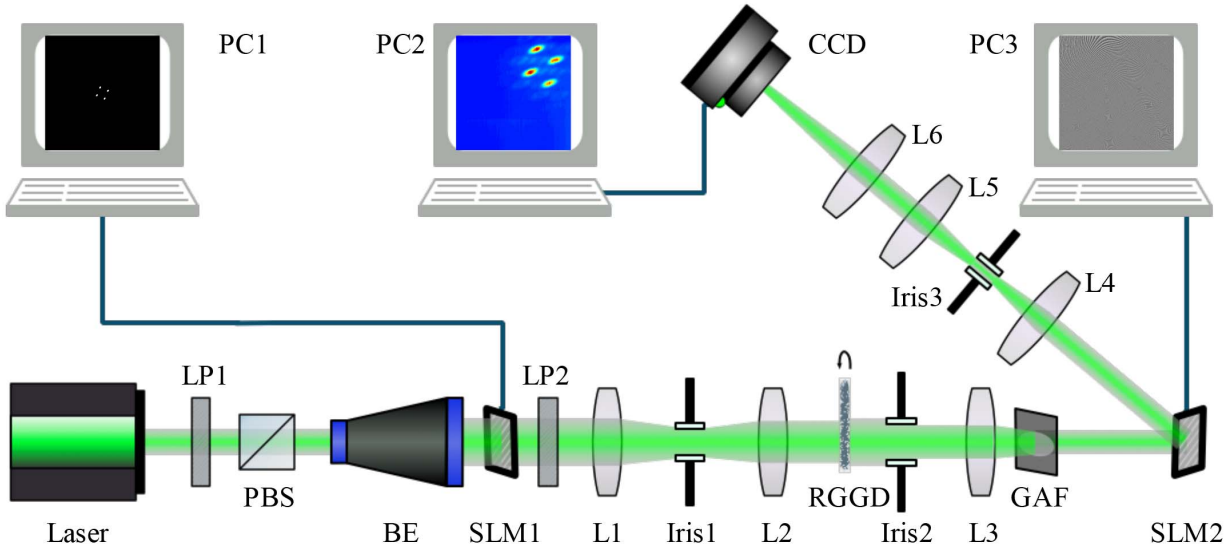

Fig. 4. Experimental setup for generating an ARGSMA beam. LP, linear polarizer; PBS, polarizing beam splitter; BE, beam expander; SLM, spatial light modulator; L, lens; RGGD, rotating ground-glass disk; GAF, Gaussian amplitude filter; CCD, charge-coupled device; PC, personal computer.

does not rotate. From $\pi / 4$ to $3 \pi / 4$, it rotates clockwise. During 0 to $\pi / 4$ and $3 \pi / 4$ to $\pi$, the rotation is counterclockwise. Then, it takes $\pi$ as the cycle to repeat itself ad infinitum. There are five special values given in Fig. 3(b), in which $\theta=2 \pi / 3$ is in perfect agreement with the transmission process in Fig. 1. Also, the corresponding points of the special values in Fig. 3(b) have been clearly marked in the periodic graph in Fig. 3(c) in order to illustrate the generality of the conclusion.

\section{Experiment}

The sketch of the experimental setup is shown in Fig. 4. The Gaussian beam is emitted by a $532 \mathrm{~nm}$ linearly polarized semiconductor laser, and it then passes through a polarizing beam splitter (PBS) to control the power of the beam. In order to

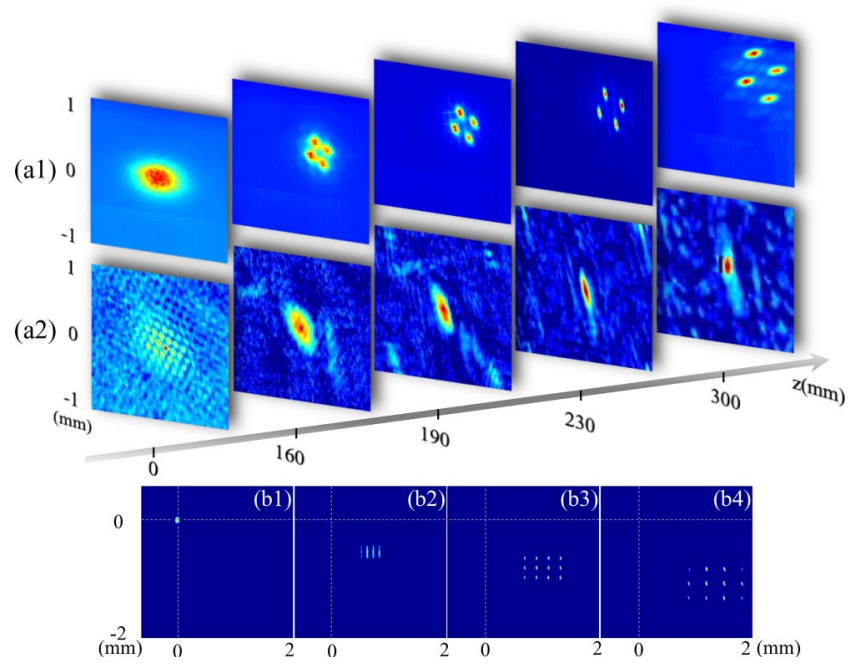

Fig. 5. (a1) Spectral density and (a2) DOC of the ARGSMA beam during transmission from the experiment and the parameters as in Fig. 1. (b1)(b4) Experimental results of free movement with $\left|\eta_{s}\right|$ increasing and the parameters as in Fig. 2. achieve the amplitude of the modulated beam, SLM1 must be distributed with a prescribed intensity. So by combining two linear polarizers (LP1 and LP2), the polarization direction of the beam after passing through the beam expander and facing SLM1 (LC2012) is easily controlled. For the purpose of filtering out stray lights, a $4 f$ system (L1 and L2) with an iris is established. After the laser beam passes through the rotating ground-glass disk (RGGD), the GSM correlation is generated $^{[23,24]}$. At the same time, other orders of light beams can be physically blocked by the iris. Then, the light beam passes through the lens after passing through the lens of focal length $f$. A Gaussian amplitude filter (GAF) is added after the lens to standardize the initial anisotropic Gaussian intensity distribution. It is also easy to know that the Fourier transform of the light intensity distribution on RGGD is equivalent to the coherent distribution formed after GAF. The next SLM2 (PLUTO, Phase Only) is loaded with the phase. After being reflected by SLM2 and going through the $4 f$ system (L4 and L5) with an iris, the anisotropic GSM beam loaded with phase hologram properties is formed in the first-order diffraction. Finally, the ARGSMA beam is generated by Fourier transform with lens (L6), and then the image is detected by the charge-coupled device (CCD) to measure the intensity of the beam. Then, one can find in Refs. [6,25] the use of a large number of light intensity photos collected to obtain the detailed information of the DOC.

The experimental parameters are consistent with the simulation values, which is easy to facilitate research, comparison, and verification. It can be seen that the results of the experiment shown in Fig. 5 are in good agreement with the numerical simulations shown earlier. First of all, it can be seen from the first set of experimental graphs that the transmission behavior of the spectral density of ARGSMA and DOC is in perfect agreement with Fig. 1. Specifically, the process of lobes splitting, the revolution direction of the array controlled by $\alpha$, the rotation behavior of the lobe controlled by $\theta$, and the final rotation angle reached are in good agreement with Fig. 1(a). Similarly, the deflection direction and angle of the DOC correspond well to 
Fig. 1(b). Subsequently, in order to give a better understanding of the dispersion mechanism of the array, the experimental diagram in Figs. 5(b1)-5(b4) verifies the process of the theoretical simulation of Figs. 2(e) to 2(i) changing with increasing $\eta$ and clearly verifies the relationship of the linear shift parameter $\eta$ with different values, which are positive or negative, and the lobes, which change in position and distance.

\section{Conclusions}

In summary, we have modeled a new family of random sources described by a revolving CSD being a linear combination of the CSDs with rotation that radiates far fields with array-like spectral densities and non-trivial distributions. Analysis shows that this type of beam can control source parameters to arbitrarily tailor revolution of the array beam and rotation of each lobe, gaining larger freedom in the field of rotary engineering. Furthermore, we demonstrated how the far-field spectra, and also found that the dimensions of the arrays, can be easily adjusted by changing the summing index in the function. By combining the construction of a partially coherent beam and the SLM loading phase, this type of ARGSMA beam is produced experimentally. Consistent with expectations, the experimental phenomena and numerical simulations basically fit. We foresee a broad range of applications for this new class of rotating beams from optical trapping and conveying, where the beam rotates and plays significant roles. More importantly, we hope that the results obtained here can realize the broadening of the field of asymmetric coherent gratings and lattices and have further enlightening research.

\section{Acknowledgement}

This work was supported by the National Natural Science Foundation of China (Nos. 12174338 and 11874321).

\section{References}

1. F. Gori and M. Santarsiero, "Devising genuine spatial correlation functions," Opt. Lett. 32, 3531 (2007).
2. S. Sahin and O. Korotkova, "Light sources generating far fields with tunable flat profiles," Opt. Lett. 37, 2970 (2012).

3. L. Wan and D. Zhao, "Twisted Gaussian Schell-model array beams," Opt. Lett. 43, 3554 (2018).

4. J. Xu, K. Pan, and D. Zhao, "Random sources generating hollow array beams," Opt. Express 28, 16772 (2020).

5. C. Liang, F. Wang, X. Liu, Y. Cai, and O. Korotkova, "Experimental generation of cosine-Gaussian-correlated Schell-model beams with rectangular symmetry," Opt. Lett. 39, 769 (2014).

6. Z. Liu and D. Zhao, "Experimental generation of a kind of reversal rotating beams," Opt. Express 28, 2884 (2020).

7. O. Korotkova and X. Chen, "Phase structuring of the complex degree of coherence," Opt. Lett. 43, 4727 (2018).

8. X. Chen and O. Korotkova, "Complex degree of coherence modeling with famous planar curves," Opt. Lett. 43, 6049 (2018).

9. X. Chen and O. Korotkova, "Phase structuring of 2D complex coherence states," Opt. Lett. 44, 2470 (2019).

10. B. Sun, Z. Huang, X. Zhu, D. Wu, Y. Chen, F. Wang, Y. Cai, and O. Korotkova, "Random source for generating Airy-like spectral density in the far field," Opt. Express 28, 7128 (2020).

11. Z. Mei and O. Korotkova, "Asymmetric coherence gratings," Opt. Lett. 45, 1366 (2020).

12. Z. Mei and O. Korotkova, "Cross-spectral densities with helical-Cartesian phases," Opt. Express 28, 20438 (2020).

13. K. Sundar, R. Simon, and N. Mukunda, "Twisted Gaussian Schell-model beams," J. Opt. Soc. Am. A 10, 2017 (1993).

14. R. Borghi, F. Gori, G. Guattari, and M. Santarsiero, "Twisted Schell-model beams with axial symmetry," Opt. Lett. 40, 4504 (2015).

15. Z. Mei and O. Korotkova, "Random sources for rotating spectral densities," Opt. Lett. 42, 255 (2017).

16. F. Gori and M. Santarsiero, "Devising genuine twisted cross-spectral densities," Opt. Lett. 43, 595 (2018).

17. R. Borghi, "Twisting partially coherent light," Opt. Lett. 43, 1627 (2018).

18. L. Wan and D. Zhao, "Controllable rotating Gaussian Schell-model beams," Opt. Lett. 44, 735 (2019).

19. K. Pan, J. Xu, and D. Zhao, "Partially coherent sources with the combined quadratic phase," Opt. Commun. 478, 126392 (2021).

20. E. Wolf, Introduction to the Theory of Coherence and Polarization of Light (Cambridge University, 2007).

21. S. A. Collins, "Lens-system diffraction integral written in terms of matrix optics," J. Opt. Soc. Am. 60, 1168 (1970).

22. Q. Lin and Y. Cai, "Tensor ABCD law for partially coherent twisted anisotropic Gaussian-Schell model beams," Opt. Lett. 27, 216 (2002).

23. M. Rousseau, "Statistical properties of optical fields scattered by random media. Application to rotating ground glass," J. Opt. Soc. Am. 61, 1307 (1971).

24. M. Mitchell, Z. Chen, M. Shih, and M. Segev, "Self-trapping of partially spatially incoherent light," J. Apl. Phys. 77, 490 (1996).

25. Y. Cai, Y. Chen, and F. Wang, "Generation and propagation of partially coherent beams with nonconventional correlation functions: a review," J. Opt. Soc. Am. A 31, 2083 (2014). 